\title{
Position Paper on Post-Traumatic Headache: The Relationship Between Head Trauma, Stress Disorder, and Migraine
}

\author{
Giorgio Lambru (D) - Silvia Benemei · Anna P. Andreou • \\ Michelangelo Luciani · Gianluca Serafini · Antoinette Maassen van den Brink • \\ Paolo Martelletti
}

Received: October 12, 2020 / Accepted: November 16, 2020 / Published online: November 28, 2020

(c) The Author(s) 2020

\begin{abstract}
Traumatic brain injury (mTBI) is a major public health concern, with mild TBI (mTBI) constituting the vast majority of the injuries. Posttraumatic headache (PTH) is one of the most frequent symptoms that follow a mTBI, occurring in isolation with a tension-type or migraine phenotype, or more often as part of a complex neurobehavioural array of symptoms. The existence of PTH as a separate entity from the primary headaches is still a matter of debate. Classification issues and a lack of methodologically robust epidemiological and clinical studies have made it difficult to elucidate the mechanisms underlying acute and even more
\end{abstract}

G. Lambru $(\bowtie) \cdot$ A. P. Andreou

The Headache Service, Pain Management and

Neuromodulation Centre, NHS Foundation Trust, Guy's and St Thomas, London, UK

e-mail: giorgio.lambru@gstt.nhs.uk

\section{S. Benemei}

Health Sciences Department, Careggi University Hospital, University of Florence, and Headache Centre, Florence, Italy

\section{A. P. Andreou}

Headache Research, Wolfson CARD, Institute of Psychiatry, Psychology and Neuroscience, King's College London, London, UK

M. Luciani · P. Martelletti

Department of Clinical and Molecular Medicine, Sapienza University, Rome, Italy persistent PTH (PPTH). Furthermore, psychiatric comorbidities such as post-traumatic stress disorder (PTSD), previous history of migraine, and legal issues often reported by PPTH patients have complicated the understanding of this condition, hence treatment approaches for PTH remain problematic. Recent findings from structural and functional neuroimaging studies have attempted to describe the brain architecture of PPTH, suggesting the involvement of different networks compared to migraine. It also seems that calcitonin gene-related peptide (CGRP) levels are not particularly raised in PPTH, although CGRP monoclonal antibodies have obtained positive initial open-label evidence of efficacy in PPTH, and more trials

M. Luciani · P. Martelletti

Emergency Medicine Unit, Regional Referral

Headache Centre, DAI Medical Sciences,

Sant'Andrea Hospital, Rome, Italy

G. Serafini

Department of Neuroscience, Rehabilitation, Ophthalmology, Genetics and Maternal Childhood Sciences, Psychiatry Unit, University of Genoa, Genoa, Italy

G. Serafini

IRCCS Ospedale Policlinico San Martino, Genoa, Italy

A. M. van den Brink

Division of Vascular Medicine and Pharmacology, Erasmus University Medical Center, Rotterdam, The Netherlands 
assessing the efficacy of this class of treatments are underway. The broad overlap between PTH, migraine, and PTSD suggests that research in this field should start with a re-appraisal of the diagnostic criteria, followed by methodologically sound epidemiological and clinical studies. Preclinical research should strive to create more reliable PTH models to support human neuroimaging, neurochemical, and neurogenetic studies, aiming to underpin new pathophysiological hypotheses that may expand treatment targets and improve the management of PTH patients.

Keywords: Migraine; Post-traumatic headache; Post-traumatic stress disorder; Tension-type headache; Traumatic brain injury

\section{Key Summary Points}

Traumatic brain injury is a growing public health concern constituting a major cause of worldwide death and disability.

Post-traumatic headache is considered a secondary headache but epidemiology, classification, and pathophysiology need clarification.

The boundaries between post-traumatic headache, post-traumatic stress disorder, and migraine present overlaps with unclear relationships.

Preclinical and clinical models of posttraumatic headache seem to confirm the recognition of post-traumatic headache as an independent entity.

Translational applications of migraine treatment to pure post-traumatic headache is premature.

\section{DIGITAL FEATURES}

This article is published with digital features, including a summary slide, to facilitate understanding of the article. To view digital features for this article go to https://doi.org/10.6084/ m9.figshare.13234526.

\section{INTRODUCTION}

\section{Background}

Traumatic brain injury (TBI) is a growing public health concern, with severe and moderate TBI constituting a major cause of worldwide death and disability $[1,2]$. Thankfully, $80 \%$ of TBIs are mild and leave subjects with minor or no cerebral damage. Nonetheless, the consequences of mild TBIs (mTBI) are often characterized by a broad array of somatosensory and cognitive symptoms, called post-traumatic syndrome, which tend to be overlooked by the public as a whole, despite causing vast medical and socioeconomic problems worldwide [3, 4], hence the term "silent public health epidemic".

Post-traumatic headache (PTH) is one of the most frequent physical symptoms that follow a mTBI, which can occur in isolation, or more often as part of a complex neurobehavioural clinical spectrum that encompasses cognitive deficits, psychiatric symptoms and the so-called post-traumatic syndrome $[5,6]$. The International Classification of Headache Disorders (ICHD-3) considers any headache starting/worsening after a TBI as attributable to the head injury if the headache occurs within 7 days from the head trauma, and subdivides them into acute and persistent according to the duration of the headache [7]. Despite being an established disorder, PTH is a topic that recognizes several complicated elements that have thus far prevented substantial advances in its understanding. 


\section{Lack of Agreement in Terminology and Limited Evidence}

These classification criteria are arbitrary and aim to stimulate research in the field, which is growing, albeit still at the initial stages. Indeed, several epidemiological and clinical key aspects of this entity need elucidation: PTH is considered a secondary headache; however, the "causative" disorder cannot be eliminated by definition, making it clinically impossible to establish a clear link between the headache and the injury. Furthermore, there is an inversely proportionate relationship between persistent post-traumatic headache and severity of the TBI, which is puzzling, although tentative explanatory hypotheses have been suggested $[8,9]$. The short temporal gap [7 days] between the TBI and onset/worsening of headache as well as the 3-month headache duration before the concept of persistency is introduced, have attempted to generate a rigid diagnostic framework that may work as the basis for novel research trying to characterize this disorder further. However, up until now, the attempt to advance our understanding of the epidemiology, clinical presentation, neurobiology, and in turn treatments, of this disorder have been hindered by the difficulty in setting up methodologically sound studies. This is due to potential limitations, namely recall bias for retrospective studies and lack of control groups and the selection of hospital-based populations for prospective studies, to list a few examples from the published literature on the topic [10].

\section{Epidemiological and Clinical Similarities with Migraine in the Absence of Trauma}

PTH is an umbrella term that often displays heterogenous headache phenotypes including a migraine-like with and without medication overuse headache $(\mathrm{MOH})$, tension-type-like phenotypes, and less frequently cervicogenic headache $[8,11]$. Trigeminal autonomic cephalalgia-like phenotypes have seldom been described [12-14]. In view of the frequent expression of a migraine phenotype after a $\mathrm{mTBI}$, a line of research hypothesizes that PTH may only constitute an exacerbation of pre-existing primary headache disorders. In this scenario, the head injury acts as a stressful precipitating/worsening factor but without any causative role [15]. This hypothesis is supported by the documented increased risk of developing persistent PTH (PPTH) in female subjects and those with a previous history of primary headache, often migraine $[16,17]$, as well as by the high prevalence of post-traumatic stress disorder (PTSD), anxiety, and depression diagnoses in patients with persistent PTH [18]. From this complex clinical picture, a mTBI would act as a mere trigger, similarly to any other chronic headache precipitating/worsening factors including family/work stressors, which are often reported by migraine patients at the start of their chronic daily symptoms, which then become more frequently persistent and difficult to treat in the subgroup of patients with preexisting psycho-pathological conditions or other sleep and/or pain comorbidities. In this scenario, persistent PTH with a migraine phenotype, often diagnosed as post-traumatic syndrome, seems clinically very similar to chronic migraine $(\mathrm{CM})$ or new daily persistent headache (NDPH) migraine variant, which are also often associated by behavioural, psychiatric, sleep, vestibular, and pain comorbidities [19, 20]. Another proposed hypothesis based on a retrospective population-based study suggests that PTH may exist as an independent clinical entity, and constitutes a subgroup of chronic daily headache [21].

\section{Limited Understanding of Underlying Mechanisms}

The pathophysiology of PTH is poorly understood. Experimental head injury models have shown early post-traumatic alterations of local nerve fibres, reduced cerebral circulation, and abnormal release of local neurotransmitters (including calcitonin gene-related peptide [CGRP], substance $P$, and vasoactive intestinal peptide [VIP]), suggesting a local neuroinflammatory process. These local processes may trigger a complex cascade of neurometabolic and neuromodulatory changes in subjects with 
persistent symptoms, which may contribute to the chronicity of the symptoms [22]. Unfortunately, it is unclear whether these changes are specific for PTH. An attempt to further characterize brain changes in PTH using neuroimaging techniques has gathered promising insights on white matter tract integrity, functional connectivity, structural volumetric changes, cerebral blood flow, and metabolism, as well as resting-state functional connectivity in acute and persistent PTH, all of which point towards the occurrence of structural and functional brain changes in PTH [23]. Furthermore, magnetic resonance imaging (MRI) studies found certain cerebral volumetric differences in painprocessing regions between patients with persistent PTH and patients with CM, creating the basis for further research to clarify the specificity of these brain changes [24, 25].

\section{Emotional and Psychological Aspects}

In general, migraine, PPTH, and PTSD have similar characteristics and need to be comprehensively addressed by clinicians in order to improve clinical outcomes. Despite the frequent overlapping between these different conditions, clinical models do not completely clarify the nature of the relations between head trauma, stress disorder, and migraine.

Exposure to a traumatic event, physical or psychological, results in the conditioning of fear and anxiety, often but not exclusive, to cues that were present at the time of the trauma, and subsequently to intense distress [26]. Trauma-related stress and anxiety involve at least three partially independent response systems: cognitive, physiological, and behavioural, and individuals will differ in how they experience and express their anxiety and distress reactions. Over the years, a number of different etiological models (e.g., based on biological, psychological, cognitive, and behavioural perspectives) tried to explain the development of PTSD [27]. These often-overlapping models do not completely explain the origin of PTSD, and often their implications for treatment are questioned. Further work is needed to disentangle the interrelationships among these factors and elucidate the underlying mechanisms [28]. Of interest, nitric oxide and endocannabinoids, which have both been implicated in migraine pathophysiology [29, 30], have been proposed to be involved in the development of PTSD [31, 32]. According to longitudinal studies, traumatic brain injury may be considered a strong predictor of both headaches and psychiatric comorbidities, and headache symptoms are prevalent after head traumas, particularly in the first 12 months after injury $[8,33]$. Importantly, a pre-existing headache disorder may be enhanced by many clinical factors such as head traumas, stress and major depression, PTSD, and environmental stressors, especially when it co-exists with deprivation and/or risk factors such as deployment [34]. Additional factors associated with persistent post-traumatic brain injury symptoms are cumulative stressors and alterations in coping strategies $[35,36]$. To this specific regard, the importance of headaches linked to trauma, aura, opioid abuse/dependence, and comorbid psychopathology, which were found to be generally associated with negative outcomes, cannot be ignored [37].

The aim of this position paper is to highlight the controversies in PTH terminology, epidemiological, clinical, and treatment data and to critically review its pathophysiological hypotheses and basic science models, with a view of directing and inspiring future development in PTH research.

This article is based on previously conducted studies and does not contain any new studies conducted by any of the authors.

\section{BASIC SCIENCE PRECLINICAL MODELS}

Animals models are indispensable for the study of many pathophysiological processes, despite the fact that an increasing range of in vitro techniques are currently available for biomedical research [38]. Investigations in the field of pain are particularly challenging, considering both the subjective nature of pain and obvious ethical concerns [39]. However, since no biochemical or genetic markers are available that 
would enable in vitro models to predict pain occurrence and evolution, as well as response to drugs, animal models are still essential in pain studies, including the study of post-traumatic headache. The headache models used in the headache research field are referred to as migraine models, although none of these fully reflects the human symptomatology. These models can be largely categorized as (a) activation of the trigeminovascular system models that imitate activation of the pain pathway, (b) infusion of nitric oxide donors, which have been shown to induce migraine in a subset of migraine patients, and (c) induction of cortical spreading depression, which is thought to represent the biological correlate of migraine aura [40].

Animal models of post-traumatic brain injury are often non-standardized and classified as mild, moderate, and severe depending on the alleged severity of injury. Because of their diversity, potentially promising neuroprotective drugs which were identified as being effective in animal TBI models have failed in phase II and III clinical trials. A more recent attempt to classify models based on the type of injury was made by Ma et al. [41], which subdivides them into focal injury, diffuse injury, and mixed injury.

Studies on PTH in animals have been based on the induction of mild traumatic brain injury. It is important to highlight that currently there are no well-established specific models for posttraumatic headache. Models of mild traumatic brain injury may be divided into penetrative and non-penetrative injuries. Penetrative brain injuries may be provoked using two different methods: (i) controlled cortical impact injury, realized using a pneumatic impactor that hits the cortex through a unilateral craniotomy [42], and (ii) lateral fluid percussion injury, where brain injury is induced by the generation of a pulse of pressurized fluid to the intact dura mater through a craniotomy [43]. Non-penetrative brain injuries may be divided into (i) weight-drop injuries, where a projectileshaped weight with a smooth surface falls from a predetermined height and hits the head of an anesthetized animal (44), and (ii) blast injuries, where the animals are usually exposed to an explosive detonation [45]. While the non- penetrative weight drop model seems the most relevant from a translation point of view due to its minimally invasive induction, the penetrative injury models are the most commonly used in the headache field. As animals cannot verbally indicate pain, in vivo models have focused on pain-related behavioural phenotypes considered indicative of headache. Cranial hypersensitivity, measured by Von Frey filaments, is one of the read-outs used to study cephalic cutaneous allodynia, which is present in both migraine [46] and post-traumatic headache [47] and reflects sensitization of the trigeminal system [48]. Using the same methodology, also peripheral (hind paw) hypersensitivity, which represents a marker of extra-cephalic allodynia, reflecting central sensitization at a level higher than the trigeminal system, may be assessed. However, this symptom is not consistently observed in studies of migraine or post-traumatic headache, complicating its translational interpretation [49]. Besides allodynia, behavioural tests may include the study of spontaneous locomotor and exploratory activity and recognition memory, as well as the avoidance of locations where a trigger was previously administered. Besides the initial injury, administration of glyceryl trinitrate or bright light stress has been used to study susceptibility to headache triggers after previous brain injury [49]. Unfortunately, studies have not attempted to investigate activation of the trigeminal system by means of cellular recordings that could more accurately assess the onset of sensitization. Additionally, a few studies have attempted to quantitate the presence of CGRP in these models, and concluded that, similarly to migraine, CGRP is increased in the trigeminal system following traumatic brain injury. The above-mentioned models have also been employed to investigate the effect of several drugs, including sumatriptan or antibodies against calcitonin gene-related peptide (CGRP) [50]. These studies have suggested that pain-related behaviours in the mTBI model depend on peripheral CGRP $[50,51]$. This could be potentially true if the injury induces inflammatory processes leading to peripheral and central sensitization. 
It is also worth mentioning that traumatic brain injury can induce or cause the brain to be further susceptible to cortical spreading depression (CSD), a phenomenon that is associated with TBI, stroke, migraines, and seizures. Often, for the induction of the CSD model in migraine research, cortical mechanical stimulation using a needle prick is utilized, i.e. by inducing mild cortical trauma. Whereas basic science studies indicate that there may be common pathways in migraine and post-traumatic headache, especially those involving CGRP, the lack of specific animal models makes it difficult to unambiguously interpret the experimental results.

Taken together, animal models have provided relevant information on the pathophysiology of PTH, but detailed underlying mechanisms are not yet fully understood. Whereas there may be an inevitable overlap between the pathophysiological pathways involved in migraine and PTH, the evidence is currently extremely scarce. Animal models should be tailored to accurately resemble human features of this headache disorder, which would allow a systematic search of similarities and differences between migraine and PTH.

\section{CLINICAL MODELS AND OVERLAP}

PTH is considered a secondary headache where the "causative" link between the new/worsening headache and the head injury can only be postulated on the basis of a close temporal occurrence of the two events. This makes PTH a controversial entity in the headache field. Although there is sufficient epidemiological evidence confirming the existence of acute PTH, PPTH remains a debated definition. The definition of "persistence" refers to the continuation of the headache for over 3 months from the head trauma. However, no indication on the headache frequency pattern of patients with PPTH is mentioned in the International Headache Society (IHS) classification criteria [7]; hence, although patients with PPTH would theoretically be expected to experience daily or nearly daily headache, paradoxically, a new- onset headache occurring within 7 days from a head injury, occurring a few days/month only, and continuing for over 3 months would fulfil the criteria for PPTH. It is difficult to believe that an episodic headache that started after a head injury would indicate a PTH biology. It is more likely that this clinical scenario represents either a worsening of a pre-existing headache disorder caused by the stress of the trauma or a coincidental event. These conclusions were supported by the findings of a partly prospective and partly retrospective study of patients with mild head injury compared to patients with minor non-cephalic traumas. The lack of significant differences in any headache domains between concussed patients and patients with other non-cephalic traumas led the authors to postulate that PPTH may be a primary headache exacerbated by the stress of the situation [53]. PPTH does not have specific clinical features, but in the majority of cases it resembles migraine, probable migraine, and tension-type headache (TTH) [15]. Based on this evidence, an alternative explanation of PPTH could be that a head trauma triggers a brain response that manifests itself with featureless head pain (TTHlike) or featureful headache (migraine-like). In this scenario, the head injury would simply be one of the many different initiating factors. This hypothesis is strengthened by the overwhelming evidence that a history of pre-injury migraine is the most powerful and consistent predictor across studies at any time point of the PTH assessment (acute and persistent) [54].

In the general population, PTH often presents with a de novo chronic daily headache (CDH) pattern, mostly in patients with, but also in patients without, a migraine biology. PTH has been found in one study to account for $15 \%$ of $\mathrm{CDH}$ in the general population, with a lifetime risk of $\mathrm{CDH}$ which increases with increasing numbers of head and neck injuries, suggesting that head injuries may be a risk factor for $\mathrm{CDH}$ [53]. In PPTH, the headache is often part of a constellation of other symptoms classified as post-concussion syndrome, including mood, sleep, vestibular and cognitive symptoms. and fatigue. Over $40 \%$ of PPTH patients are depressed, and depression is a strong predictor for PTH [55]. Anxiety disorders may occur 
in one fourth of patients with PTH. Anxiety was also found to be linked to cognitive impairment, and depression has been found to be predictive of social anxiety in clinical traumatic head injury models [56]. Anxiety has also found to be linked to sleep disorders and fatigue. Sleep disorders, as well as fatigue, have been found to be predictors of PTH severity [57]. Cognitive impairment in PTH often presents with significant reductions in executive function, information processing speed, visual sensory input integration, and forgetfulness [58, 59]. Furthermore, approximately $30 \%$ of PPTH patients also suffer from post-traumatic stress disorder (PTSD), which is associated with further functional impairment in PPTH patients [60]. However, it is difficult to establish whether these symptoms are somehow linked to the biology of PPTH, or whether they are exacerbated by the psycho-social changes that some subjects with any chronic pain experience. Indeed, a pre-existing headache disorder may be enhanced by many factors such as head traumas, stress, major depression, and PTSD [61]. Given that the most frequent pre-existing headache disorder in PTH patients is migraine, it is possible to postulate that head trauma is a trigger/worsening factor of a pre-existing headache disorder, which is daily and causes severe disability in a subgroup of patients with a pre-existing psychiatric history and other comorbidities typically associated with chronic migraine (CM) $[62,63]$. There is a direct link between multiple comorbidities and progression to $\mathrm{CM}$ with associated high levels of headache-related disability [20], suggesting a clinical overlap between PPTH and CM. A de novo chronic continuous daily headache triggered by a specific event may also suggest that PPTH constitutes a form of new daily persistent headache (NDPH). In NDPH, a remitting and a persistent form have been identified, and in idiopathic cases, a TTH and a migraine variant have been described. Similarly to PPTH, severe anxiety and depression and other psychiatric comorbidities have been reported in patients with NDPH [19]. Similar to PPTH, NDPH in many cases is difficult to treat, suggesting perhaps a clinical and pathophysiological overlap between these two $\mathrm{CDH}$ forms and refractory $\mathrm{CM}$.
The pathophysiology of PTH is unknown. Postulated mechanisms for PTH include local biochemical changes, including excessive release of excitatory amino acids and nitric oxide, alterations in neurotransmitter functioning (namely 5-HT), disturbances in vasoactive peptides and endogenous opioids, magnesium deficiency, and loss of calcium homeostasis [64]. The persistence of the headache over 3 months may occur when central sensitization mechanisms intervene [65]. These include impaired descending inhibitory modulation of the trigeminocervical complex, impaired cortical excitability, and neuroinflammatory changes [66]. Preliminary data have also suggested that increased levels of CGRP are observed in brain injury animal models. CGRP antagonist and sumatriptan were able to mitigate these increased levels, suggesting a potential role of CGRP-dependent mechanisms in acute PTH [67]. This finding may support the use of CGRP modulators in the acute and preventive treatment of $\mathrm{PTH}$. If the mechanisms driving the persistent head pain in PPTH are shared with those of $\mathrm{CM}$, then it is likely that a subgroup of patients with PPTH with a CM phenotype will benefit from these approaches [68].

Interestingly, neuroimaging techniques looking at functional and structural brain differences between PPTH and CM subjects have highlighted some differences, which may support the presence of different pathophysiological substrates between PPTH and CM, and point towards the recognition of PPTH as an independent entity [24, 25]. However, these findings may be biased by factors like different patient selection (difference in the duration of the condition in years, number of monthly migraine as opposed to headache days, and the presence of a daily continuous pattern as opposed to a non-daily pattern), presence of comorbidities (psychiatric, sleep, non-cephalic pain), and level of treatment refractoriness (naïve to preventive treatments vs difficult-totreat patients). All these factors may play a role in functional brain networks and volumetric regional changes, suggesting caution in the interpretation and generalization of these results. Indeed, diffusion tensor imaging 
evidence in subjects with PTH and PPTH with a migraine phenotype versus subjects with mild TBI and no subsequent headache showed MRI changes which overlap with the ones found in migraine, suggesting common underlying mechanisms between PPTH and migraine [69].

\section{TREATMENT}

The uncertainty on the neurobiology of PTH has limited any significant research advancements on effective treatments. The clinical impression is that PPTH is largely a difficult-totreat condition, although the reasons remain unknown. Factors that may play a role may include the head trauma characteristics, gender, previous personal headache history, presence of PTSD, other mental health conditions and other comorbidities, and the presence of legal issues. The lack of understanding on whether these factors are relevant and to what degree they influence the response to treatments has hindered the development of specific treatment approaches for this condition. Furthermore, understanding the treatment refractoriness mechanisms would be pivotal to explain why a significant proportion of PPTH patients end up becoming resistant to treatments. Given that the PPTH phenotype shares clinical and management strategies with NDPH and CM, which are also frequently difficult to treat and associated with psychopathological comorbidities, shared mechanisms may account for the treatment refractoriness of these disorders, the elucidation of which would lead to a major advancement in the field.

Instead, in clinical practice the acute and preventive management of PPTH is based on the clinical phenotype, which often mimics that of primary headache disorders. Pharmacological treatments used in migraine have been tried in PPTH with mixed results [70]. Injectable treatments, namely occipital nerve blocks and onabotulinum toxin A, hold positive open-label evidence [71]. Noninvasive and invasive neuromodulation approaches may also have a role in PPTH treatment. Repetitive transcranial magnetic resonance (rTMS) was shown to lead to favourable outcomes in terms of headache characteristics and headache-related quality of life [72]. PTH is often associated with a cervicogenic headache, possibly due to a whiplash injury component. In such patients, invasive neuromodulation, namely occipital nerve stimulation (ONS), has shown promising clinical outcomes [73]. However, the methodology of these studies is generally poor, and no randomized controlled trials have yet been produced.

The role of CGRP monoclonal antibodies in PTH is being studied. Initial open-label shortterm evidence on the effectiveness of erenumab in PPTH with a predominant CM phenotype showed a meaningful response in $28 \%$ of the patients, which is lower than the responder rate that emerged from the CM studies in difficultto-treat populations [74-76]. Fremanezumab is being studied for the treatment for PTH in a phase 2 multicentre randomized controlled study (ClinicalTrials.gov Identifier: NCT03347188). CGRP infusion has been shown to induce a migrainous headache in PPTH subjects after a mTBI [77]. However, when measured from the venous blood, CGRP plasma levels were lower in PPTH subjects [78], suggesting that more research will have a crucial goal to clarify whether the CGRP nociceptive pathway involvement reflects a disease-specific mechanism in PPTH or an aspecific involvement of the trigeminovascular system.

Following the positive evidence in $\mathrm{CM}$, different subtypes of botulinum toxin A are being studied in PTH. Prospective studies testing the efficacy of onabotulinum toxin A and abobotulinum toxin A have been recently completed (ClinicalTrials.gov Identifiers: NCT02160535 and NCT03928496). Psilocybin belongs to the class of organic compounds known as tryptamines and derivatives. It is being studied in a small phase 2 study at two different doses versus placebo (ClinicalTrials.gov Identifier: NCT03806985). These treatments may well be effective in ultimately improving patients' quality of life, and hopefully stimulating further clinical, biochemical, and neuroimaging research in the field. Treating certain symptoms, namely the migraine ones, without a clear understanding of the full underlying neurobiological picture may only treat one of 
the disorder's clinical manifestations, not the condition as a whole. Similarly, treating the $\mathrm{MOH}$, the mental health, or the non-cephalic pain aspects in isolation may not produce the expected treatment outcome. The management of PPTH should be multidisciplinary, involving headache specialists, headache nurses, psychiatrists/psychologists, pain specialists, and occupational therapists, to tackle the multiple facets of the condition. Pharmacological treatments should be selected according to the phenotype and comorbidities. Quite often, given the severity and impact of mood disorders, a psychiatric assessment and treatment including antidepressants/anti-anxiety medications and PTSD counselling need to be combined with headache prophylaxis. Often, pharmacological treatments need to be combined with injectable or neuromodulation approaches to restore some quality of life. When expensive treatments are offered, it is important to address any pending legal issues first, which notoriously prevent full treatment effectiveness.

\section{CONCLUSION}

The debate on the relationship between mTBI, migraine, PTH, and PTSD has not yet found a definitive position $[79,80]$. It is unclear whether a head trauma triggers a biologically unique headache condition or whether the head pain mechanisms share migraine or tension-type pathways. Furthermore, it is possible that a head trauma is just a trigger of a preexisting headache disorder, which becomes persistent in a subgroup of subjects with certain genetic characteristics, exposed to certain environmental factors. In this contest, the presence of co-morbidities may contribute to render patients refractory to medical treatments, similarly to what is seen in clinical practice for refractory $\mathrm{CM}$ patients. [81]. In the absence of any study confirming whether PPTH is a separate entity or merely an exacerbation of pre-existing migraine, tension-type headache, or a subform of NDPH, neuroimaging research has been the first attempt to objectively differentiate between PPTH and migraine, though data are still preliminary and somewhat conflicting. In this scenario, the hypothesis of administration of innovative drugs may therefore be premature. The terminology issues and lack of specificity of the ICHD-3 diagnostic criteria for PTH constitute the main limitation for any major advancement in the field. Basic scientists and clinicians should collaborate towards the development of specific animal models of PTH along with biochemical and neuroimaging studies in humans to confirm the existence of PTH as a separate entity. Once established, then prospective population-based epidemiological studies, along with studies assessing headache persistency and refractoriness mechanisms, may set the scene for the development of mechanism-specific novel treatments, for which a vast unmet need exists at present.

\section{ACKNOWLEDGEMENTS}

Funding. No funding or sponsorship was received for this study or publication of this article.

Authorship. All named authors meet the International Committee of Medical Journal Editors (ICMJE) criteria for authorship for this article, take responsibility for the integrity of the work as a whole, and have given their approval for this version to be published

Disclosures. Giorgio Lambru, Silvia Benemei, Anna P. Andreou, Michelangelo Luciani, Gianluca Serafini and Antoinette Maassen van den Brink have nothing to disclose. Paolo Martelletti is a member of the journal's Editorial Board.

Compliance with Ethics Guidelines. This article is based on previously conducted studies and does not contain any new studies conducted by any of the authors.

Data Availability. Data sharing is not applicable to this article as no datasets were generated or analysed during the current study. 
Open Access. This article is licensed under a Creative Commons Attribution-NonCommercial 4.0 International License, which permits any non-commercial use, sharing, adaptation, distribution and reproduction in any medium or format, as long as you give appropriate credit to the original author(s) and the source, provide a link to the Creative Commons licence, and indicate if changes were made. The images or other third party material in this article are included in the article's Creative Commons licence, unless indicated otherwise in a credit line to the material. If material is not included in the article's Creative Commons licence and your intended use is not permitted by statutory regulation or exceeds the permitted use, you will need to obtain permission directly from the copyright holder. To view a copy of this licence, visit http:// creativecommons.org/licenses/by-nc/4.0/.

\section{REFERENCES}

1. Dewan MC, et al. Estimating the global incidence of traumatic brain injury. J Neurosurg. 2018;27:1-18.

2. Langlois JA, Rutland-Brown W, Wald MM. The epidemiology and impact of traumatic brain injury: a brief overview. J Head Trauma Rehabil. 2006;21: 375-8.

3. Yilmaz T, et al. Risk factors and outcomes associated with post- traumatic headache after mild traumatic brain injury. Emerg Med J. 2017a;34:800-5.

4. Bedaso A, Geja E, Ayalew M, Oltaye Z, Duko B. Postconcussion syndrome among patients experiencing head injury attending emergency department of Hawassa University Comprehensive specialized hospital, Hawassa, southern Ethiopia. J Headache Pain. 2018;19:112.

5. Lucas S, Hoffman JM, Bell KR, Dikmen S. A prospective study of prevalence and characterization of headache following mild traumatic brain injury. Cephalalgia. 2014;34:93-102.

6. Minen MT, Boubour A, Walia H, Barr W. Post-concussive syndrome: a focus on post-traumatic headache and related cognitive, psychiatric, and sleep issues. Curr Neurol Neurosci Rep. 2016;16:100.

7. Headache Classification Committee of the International Headache Society (IHS). The International
Classification of Headache Disorders, 3rd edition. Cephalalgia. 2018;38:1-211.

8. Lucas S, Hoffman JM, Bell KR, Walker W, Dikmen S. Characterization of headache after traumatic brain injury. Cephalalgia. 2012;32:600-6.

9. Packard RC. Chronic post-traumatic headache: associations with mild traumatic brain injury, concussion, and post-concussive disorder. Curr Pain Headache Rep. 2008;12:67-73.

10. Couch JR, Lipton R, Stewart WF. Is post-traumatic headache classifiable and does it exist? Eur J Neurol. 2009;16:12-3.

11. Samim F, Epstein JB. Orofacial neuralgia following whiplash-associated trauma: case reports and literature review. SN Compr Clin Med. 2019;1:627-32.

12. Lambru G, Castellini P, Manzoni GC, Torelli P. Post-traumatic cluster headache: from the periphery to the central nervous system? Headache. 2009;49:1059-61.

13. Lambru G, Rantell K, Levy A, Matharu MS. A prospective comparative study and analysis of predictors of SUNA and SUNCT. Neurology. 2019;93: e1127-37.

14. Matharu MJ, Goadsby PJ. Post-traumatic chronic paroxysmal hemicrania $(\mathrm{CPH})$ with aura. Neurology. 2001;56:273-5.

15. Haas DC. Chronic post-traumatic headaches classified and compared with natural headaches. Cephalalgia. 1996;16:486-93.

16. Hoffman JM, et al. Natural history of headache after traumatic brain injury. J Neurotrauma. 2011;28: 1719-25.

17. Yilmaz T, et al. Risk factors and outcomes associated with post- traumatic headache after mild traumatic brain injury. Emerg Med J. 2017b;34:800-5.

18. Chibnall JT, Duckro PN. Post-traumatic stress disorder in chronic post-traumatic headache patients. Headache J Head Face Pain. 1994;34:357-61.

19. Uniyal R, Paliwal VK, Tripathi A. Psychiatric comorbidity in new daily persistent headache: a cross-sectional study. Eur J Pain. 2017;21:1031-8.

20. Lipton RB, Fanning KM, Buse DC, Martin VT, Hohaia LB, Adams AM, Reed ML, Goadsby PJ. Migraine progression in subgroups of migraine based on comorbidities: Results of the CaMEO Study. Neurology. 2019;93:e2224-36.

21. Couch JR, Lipton RB, Stewart WF, Scher AI. Head or neck injury increases the risk of chronic daily 
headache: a population-based study. Neurology. 2007a;69:1169-77.

22. Packard RC. Epidemiology and pathogenesis of posttraumatic headache. J Head Trauma Rehabil. 1999;14:9-21.

23. Schwedt TJ. Structural and functional brain alterations in post-traumatic headache attributed to mild traumatic brain injury: a narrative review. Front Neurol. 2019;10:615.

24. Schwedt TJ, Chong CD, Peplinski J, Ross K, Berisha V. Persistent post-traumatic headache vs. migraine: an MRI study demonstrating differences in brain structure. J Headache Pain. 2017;18:87.

25. Dumkrieger G, Chong CD, Ross K, Berisha V, Schwedt TJ. Static and dynamic functional connectivity differences between migraine and persistent post-traumatic headache: a resting-state magnetic resonance imaging study. Cephalalgia. 2019;39: 1366-81.

26. Zoellner LR, Eftekhari A, Bedard-Gilligan M. Psychological Models of Posttraumatic Stress Disorder and Acute Stress Disorder. Oxford Handbook of Anxiety and Related Disorders; Edited by Martin M. Antony M.M; Stein M.B. 2008.

27. Jones JC, Barlow DH. The etiology of posttraumatic stress disorder. Clin Psychol Rev. 1990;10:299-328.

28. Keane TM, Marshall AD, Taft CT. Posttraumatic stress disorder: etiology, epidemiology, and treatment outcome. Ann Rev Clin Psychol. 2006;2: 161-97.

29. Andreou AP, Edvinsson L. Mechanisms of migraine as a chronic evolutive condition. J Headache Pain. 2019;20:117.

30. Lambru G, Andreou AP, Guglielmetti M, Martelletti P. Emerging drugs for migraine treatment: an update. Expert Opin Emerg Drugs. 2018;23:301-18.

31. Neumeister A, Seidel J, Ragen BJ, Pietrzak RH. Translational evidence for a role of endocannabinoids in the etiology and treatment of posttraumatic stress disorder. Psychoneuroendocrinology. 2015;51:577-84.

32. Pall ML, Satterlee JD. Elevated nitric oxide/peroxynitrite mechanism for the common etiology of multiple chemical sensitivity, chronic fatigue syndrome, and posttraumatic stress disorder. Ann N Y Acad Sci. 2001;933:323-9.

33. Jaramillo CA, Eapen BC, McGeary CA, Robinson J, Amuan M, Pugh MJ. A cohort study examining headaches among veterans of Iraq and Afghanistan wars: Associations with traumatic brain injury,
PTSD, and depression. Headache J Head Face Pain. 2015;56:528-39.

34. Theeler BJ, Flynn FG, Erickson JC. Chronic daily headache in US soldiers after concussion. Headache J Head Face Pain. 2012;52, 732-738.

35. Iverson GL. Outcome from mild traumatic brain injury. Curr Opin Psychiatry. 2005;18:301-17.

36. Wood RL. Understanding the 'miserable minority': a diasthesis-stress paradigm for post-concussional syndrome. Brain Inj. 2004;18:1135-53.

37. Cohen SP, Plunkett AR, Wilkinson I, Nguyen C, Kurihara C, Flagg A, Morlando B, Stone C, White RL, Anderson-Barnes VC, et al. Headaches during war: analysis of presentation, treatment, and factors associated with outcome. Cephalalgia. 2011;32: 94-108.

38. Barré-Sinoussi F, Montagutelli X. Animal models are essential to biological research: issues and perspectives. Future Sci. 2015;OA 1:FSO63.

39. Mogil JS. Animal models of pain: progress and challenges. Nat Rev Neurosci. 2009;10:283-94.

40. Andreou AP, Summ O, Charbit AR, Romero-Reyes M, Goadsby PJ. Animal models of headache: from bedside to bench and back to bedside. Expert Rev Neurother. 2010;10:389-411.

41. Ma X, Aravind A, Pfister BJ, Chandra N, Haorah J. Animal Models of Traumatic Brain Injury and Assessment of Injury Severity. Mol Neurobiol. 2019;56:5332-45.

42. Lighthall JW. Controlled cortical impact: a new experimental brain injury model. J Neurotrauma. 1988;5:1-15.

43. McIntosh TK, Vink R, Noble L, et al. Traumatic brain injury in the rat: characterization of a lateral fluid-percussion model. Neuroscience. 1989;28: 233-44.

45. Goldstein LE, McKee AC, Stanton PK. Considerations for animal models of blast-related traumatic brain injury and chronic traumatic encephalopathy. Alzheimers Res Ther. 2014;6:64.

46. Bigal ME, Ashina S, Burstein R, Reed ML, Buse D, Serrano D, et al. Prevalence and characteristics of allodynia in headache sufferers: a population study. Neurology. 2008;70:1525-33.

47. Markus TE, Zeharia A, Cohen YH, Konen O. Persistent headache and cephalic Allodynia attributed to head trauma in children and adolescents. J Child Neurol. 2016;31:1213-9. 
48. Burstein R, Yarnitsky D, Goor-Aryeh I, Ransil BJ, Bajwa ZH. An association between migraine and cutaneous allodynia. Ann Neurol. 2000;47:614-24.

49. Benemei S, Labastida-Ramírez A, Abramova E, et al. Persistent post-traumatic headache: a migrainous loop or not? The preclinical evidence. J Headache Pain. 2020;21:90.

50. Bree D, Levy D. Development of CGRP-dependent pain and headache related behaviours in a rat model of concussion: implications for mechanisms of post-traumatic headache. Cephalalgia. 2018;38: 246-58.

51. Navratilova E, Rau J, Oyarzo J, et al. CGRP-dependent and independent mechanisms of acute and persistent post-traumatic headache following mild traumatic brain injury in mice. Cephalalgia. 2019;39:1762-75.

52. Mickeviciene D, Schrader H, Obelieniene D, et al. A controlled prospective inception cohort study on the post-concussion syndrome outside the medicolegal context. Eur J Neurol. 2004;11:411-9.

53. Couch JR, Lipton RB, Stewart WF, Scher AI. Head or neck injury increases the risk of chronic daily headache: a population-based study. Neurology. 2007b;69:1169-77.

54. Nordhaug LH, Hagen K, Vik A, Stovner LJ, Follestad T, Pedersen T, Gravdahl GB, Linde M. Headache following head injury: a population-based longitudinal cohort study (HUNT). J Headache Pain. 2018;19:8.

55. Lieba-Samal D, Platzer P, Seidel S, Klaschterka P, Knopf A, Wober C. Characteristics of acute posttraumatic headache following mild head injury. Cephalalgia. 2011;31:1618-26.

56. Curvis W, Simpson J, Hampson N. Social anxiety following traumatic brain injury: an exploration of associated factors. Neuropsychol Rehabil. 2016;17: $1-21$.

57. Bomyea J, Lang AJ, Delano-Wood L, et al. Neuropsychiatric predictors of post-injury headache after mild-moderate traumatic brain injury in veterans. Headache. 2016;56:699-710.

58. Finnanger TG, Skandsen T, Andersson S, Lydersen S, Vik A, Indredavik M. Differentiated patterns of cognitive impairment 12 months after severe and moderate traumatic brain injury. Brain Inj. 2013;27:1606-16.

59. Starkey NJ, Jones K, Case R, Theadom A, BarkerCollo S, Feigin V. Post-concussive symptoms after a mild traumatic brain injury during childhood and adolescence. Brain Inj. 2018;32:617-26.
60. Kjeldgaard D, Forchhammer H, Teasdale T, Jensen RH. Chronic post-traumatic headache after mild head injury: a descriptive study. Cephalalgia. 2013;34:191-200.

61. Guglielmetti M, Serafini G, Amore M, Martelletti P. The relation between persistent post-traumatic headache and PTSD: similarities and possible differences. Int J Environ Res Public Health. 2020;17: 4024.

62. Labastida-Ramírez A, Benemei S, Albanese M, et al. European Headache Federation School of Advanced Studies (EHF-SAS). Persistent post-traumatic headache: a migrainous loop or not? The clinical evidence. J Headache Pain. 2020;21:55.

63. Chan TLH, Woldeamanuel YW. Exploring naturally occurring clinical subgroups of post-traumatic headache. J Headache Pain. 2020;21:12.

64. Packard RC, Ham LP. Pathogenesis of posttraumatic headache and migraine: a common headache pathway? Headache. 1997;37:142-52.

65. Leung A. Addressing chronic persistent headaches after MTBI as a neuropathic pain state. J Headache Pain. 2020;21:77.

66. Ashina H, Porreca F, Anderson T, et al. Post-traumatic headache: epidemiology and pathophysiological insights. Nat Rev Neurol. 2019;15:607-17.

67. Daiutolo BV, Tyburski A, Clark SW, Elliott MB. Trigeminal pain molecules, allodynia, and photosensitivity are pharmacologically and genetically modulated in a model of traumatic brain injury. J Neurotrauma. 2016;15:748-60.

68. Ashina H, Iljazi A, Al-Khazali HM, et al. Efficacy, tolerability, and safety of erenumab for the preventive treatment of persistent post-traumatic headache attributed to mild traumatic brain injury: an open-label study. J Headache Pain. 2020a;21:62.

69. Alhilali LM, Delic J, Fakhran S. Differences in callosal and forniceal diffusion between patients with and without Postconcussive migraine. AJNR Am J Neuroradiol. 2017;38:691-5.

70. Larsen EL, Ashina H, Iljazi A, et al. Acute and preventive pharmacological treatment of post-traumatic headache: a systematic review. J Headache Pain. 2019;20:98.

71. Conidi FX. Interventional Treatment for Post-traumatic Headache. Curr Pain Headache Rep. 2016;20: 40 .

72. Stilling J, Paxman E, Mercier L, et al. Treatment of persistent post-traumatic headache and post-concussion symptoms using repetitive transcranial 
magnetic stimulation: a pilot, double-blind randomized controlled trial. J Neurotrauma. 2020;37: 312-23.

73. Eghtesadi M, Leroux E, Fournier-Gosselin MP, et al. Neurostimulation for Refractory Cervicogenic Headache: A Three-Year Retrospective Study. Neuromodulation. 2018;21:302-9.

74. Ashina H, Iljazi A, Al-Khazali HM, et al. Efficacy, tolerability, and safety of erenumab for the preventive treatment of persistent post-traumatic headache attributed to mild traumatic brain injury: an open-label study. J Headache Pain. 2020b;21:62.

75. Ashina M, Tepper S, Brandes JL, et al. Efficacy and safety of erenumab (AMG334) in chronic migraine patients with prior preventive treatment failure: a subgroup analysis of a randomized, double-blind, placebo-controlled study. Cephalalgia. 2018;38: 1611-21.

76. Lambru G, Hill B, Murphy M, Tylova I, Andreou AP. A prospective real-world analysis of erenumab in refractory chronic migraine. J Headache Pain. 2020;21:61.
77. Ashina H, Iljazi A, Al-Khazali HM, et al. Hypersensitivity to calcitonin gene-related peptide in posttraumatic headache. Ann Neurol. 2020; Sep 21. Online ahead of print.

78. Ashina $\mathrm{H}, \mathrm{Al}-\mathrm{Kh}$ azali $\mathrm{HM}$, Iljazi A, et al. Low plasma levels of calcitonin gene-related peptide in persistent post-traumatic headache attributed to mild traumatic brain injury. Cephalalgia. 2020. Online ahead of print.

79. Capi M, Pomes LM, Andolina G, Curto M, Martelletti P, Lionetto L. Persistent post-traumatic headache and migraine: pre-clinical comparisons. Int J Environ Res Public Health. 2020;17:2585.

80. Benemei S, Labastida-Ramírez A, Abramova E, et al. Persistent post-traumatic headache: a migrainous loop or not? The preclinical evidence. European Headache Federation School of Advanced Studies (EHF-SAS). J Headache Pain. 2020;21:90.

81. Simona S, Mark B, Anne D, Christian L, Patrick L, van den Brink, AM et al. European headache federation consensus on the definition of resistant and refractory migraine. J Headache Pain. 2020 21:76. 